\title{
Pressure, Anxiety and Subjective well being in Chinese Overseas Returnees
}

\author{
Huang Xitong ${ }^{1}$, Guo Wenwen ${ }^{2}$, Fu Guifang ${ }^{1 *}$ \\ ${ }^{I}$ Department of Applied Psychology, School of Public Administration, Guangdong University of Foreign \\ Studies, Canton, China \\ ${ }^{2}$ International College, Guangdong University of Foreign Studies, Canton, China
}

*Corresponding Author: $\boldsymbol{F u}$ Guifang, Department of Applied Psychology, School of Public Administration, Guangdong University of Foreign Studies, Canton, China.

\begin{abstract}
To explore the current situation and relationship of overseas returnees' pressure, anxiety and subjective well-being, survey was used to test 331 overseas returnees from the United States, Britain, France, Germany, Japan, etc. back to China. The results showed that: (1) the pressure, anxiety and subjective wellbeing of the overseas returnees were at a medium level. (2) The anxiety of overseas returnees was significantly different in the marital status dimension, and the level of anxiety of unmarried persons was significantly higher than that of married people. (3) The pressure, anxiety subjective well being of the returned overseas people was significantly negatively correlated, and the pressure and anxiety negatively predicted subjective well-being.
\end{abstract}

Keywords: overseas returnees, pressure, anxiety, subjective well-being

\section{INTRODUCTION}

Globalization makes studying abroad more popular and necessary. Studying abroad is not only to learn professional skills, but also to experience different cultures and customs. According to the World Bank statistics data, from 1997 to 2011, the average annual growth rate of international students in the world was more than twice the growth rate of world GDP. Although students in many countries chose to stay in the host country after completing their overseas education, a new trend has emerged recently, that is, overseas students choose to return to their home country for employment or emigration. It is particularly obvious in China that more and more overseas students are returning to work, which has reversed the brain drain. The reason why overseas students choose to return home may be that they are inspired by the government, their family relations and the rapid development of China's politics and economy in the past 40 years. But now there are few studies on returned students.

Overseas researches on returnees were mainly focused on returnees' capital accumulation (Takumi Naito, Laixun Zhao, 2019) and the influencing factors of overseas students' migration (Abbott, A., silles, M, 2016), two growth effects of overseas learning (the former "brain effect" is for immigrants to study abroad, and the latter "brain loss effect" is for the actual migration of talents, Michel Beine, Frédéric Docquier, Hillel Rapoport, 2001), personnel and time for returning to study abroad and requirements for returning to study abroad (Kahn, S., Macgarvie, M, 2016). Some researchers also studied the United Front work, employment, innovative studies, cultural identity and bicultural identity, employment satisfaction, political participation of Chinese returnees and so on. But there were not many researches on the psychological aspects of returnees. Therefore, this study attempts to explore the current situation and relationship of stress, anxiety and subjective well-being of Chinese returnees.

Subjective well-being refers to people's emotional and cognitive evaluation of their quality of life and happiness perception. It is a psychological state formed by interaction with the outside world when people's ideals are realized and needs are met. Campell believes that subjective well-being reflects the balance of positive emotion and negative emotion. When positive emotion prevails over negative emotion, the subjective well-being of the individual presents a higher level (Han Haiping, 2012). A study found that the level of subjective well-being of returnees in Shanghai technology enterprises 
was lower than that of local knowledge workers, and there was a significant negative correlation between work pressure and subjective well-being (Han Haiping, 2012). At the same time, the study also found that there was a linear relationship between life satisfaction and work experience, life security and personal value, among which life security had a greater impact on the overall level (DuanHongzhen, 2015). This shows that the living conditions, quality and income security of returnees affect their good understanding of the environment. There were significant differences in gender dimensions in the subjective well-being of Returned Overseas Chinese, among which the overall subjective well-being of female knowledge workers was positively correlated with their work performance (Wu Yanmei, 2013), indicating that the positive cognition of individual happiness would have a positive effect on their work efficiency.

Stress refers to the negative feelings and beliefs that individuals feel unable to cope with environmental requirements. According to Phillip L. rice (2000), the concept of stress has three meanings: an event or environmental stimulus that makes people feel nervous; a physiological or psychological response caused by a threatening stimulus; and the interaction between stimulus and response. The stressor refers to the factors that cause individual stress response, including biological stressor, spiritual stressor and social stressor. Due to cultural differences, life adaptation, interpersonal communication and other reasons, the returnees are suffering from the dual pressure of environment and psychology.

Some researches found that the motivation of returnees included personal development, family, policy and environment, while the factors affecting their career development after returning to China mainly include environment, policy, family, personal development, scientific research and academic factors (TianHaisong, 2012). Due to their international education background, overseas returnees have high expectations for work in the process of job hunting; due to the change of environment, they are faced with the problem of cultural readjustment; the shortcomings of domestic system and environmental defects also hinder the employment and Entrepreneurship of overseas returnees (Li Liping, 2013). At the same time, the returnees bear the pressure of housing and maladjustment to management system and interpersonal communication (Yin Haoyang, 2017). In view of the impact of reverse cultural shock on returnees, it was found that the cultural adaptation of returnees in Shanghai was generally good, but there were still problems such as adjustment of life rhythm and values, employment, health, guidance and service (Li Jing, 2008). It can be seen that the returnees are facing such pressures as the deviation of environment and self-expectation, the obstacle of adaptation of working environment and cultural environment, etc.

The returnees are facing the pressure from economy, politics and culture. Their stress level is at a high level in the face of cultural adaptation, work challenges and interpersonal problems. The negative emotion of anxiety often appears when the returned students encounter the challenges and problems in life, so anxiety is a common psychological state of many returned students.

Researches found that the stress and anxiety were negatively correlated with subjective well-being, and the negative stress predicted the subjective well-being. HouRuihe et al. (2013) found that anxiety was negatively correlated with positive emotion and positively correlated with negative emotion. However, the research results of RenHuizhu on the staff showed that job anxiety played an intermediary role between job stress and job happiness (RenHuizhu, 2017). It shows that the higher the work pressure, the lower the job satisfaction and the sense of well-being; at the same time, work anxiety plays an intermediary role in the influence of work pressure on the subjective well-being of employees. Stress and anxiety are both important factors that affect individual subjective well-being. Therefore, there is great practical value to further explore the relationship between stress, anxiety and subjective well-being of Chinese returned students.

\section{METHOD}

\subsection{Participants}

This study used a questionnaire survey. A total of 331 questionnaires were distributed to those who have studied in Australia, Germany, Japan, the United States, the United Kingdom and other countries and returned to work and life. 331 valid questionnaires were recovered, with an effective rate of $100 \%$. There were 159 men and 172 women; 180 were married and 151 were single. 


\subsection{Instruments}

\subsubsection{Pressure Scale}

This study used a self-developed pressure scale to examine the overall pressure of the participants in five aspects: employment, work, life, economy, and interpersonal communication. There were 5 items in the questionnaire, using a 5-point scoring method, which was divided into 5 levels: "strongly disagree", "disagree", "general", "agree", "strongly agree". The reliability of this questionnaire was 0.867 .

\subsubsection{Anxiety Scale}

In this study, the anxiety scale in the SCL-90 scale was used as a scale to examine the anxiety status of the participants. The scale had a total of 10 items, using a 5-point scoring method, which was divided into 5 levels: "none", "mild", "moderate", "quite heavy" and "severe". The reliability of this scale was 0.943 .

\subsubsection{Index of Well-Being Scale}

The Index of well-being scale scale compiled by Camppell in 1976 was adopted, including the overall emotion index scale and the life satisfaction scale. The former consisted of 8 emotional items, and the latter consisted of a satisfaction item. The weighted sum of the scores of the two was the overall happiness index. The first part had a weight of 1.0 and the second part had a 1.1. The higher the score, the higher the degree of subjective well-being. The questionnaire used 7-point scoring. Participants chose the most suitable numerical description to describe the degree of options that were most suitable for them. In this study, the overall internal consistency coefficient ( $\alpha$ coefficient) of the scale was 0.825

\subsection{Statistical Analysis}

This study used SPSS 20.0 and M Plus for Windows 7.4 statistical software for statistical analysis of the data.

\section{RESULTS}

\subsection{Reliability and Factor Analysis of Each Scale}

Table1. Reliability of each scale (Cronbach $\alpha$ coefficient)

\begin{tabular}{|l|l|l|l|}
\hline$n$ & Pressure Scale & Anxiety Scale & Index of well-being Scale \\
\hline 331 & 0.867 & 0.943 & 0.825 \\
\hline
\end{tabular}

After reliability analysis, the reliability of the pressure scale, anxiety scale and subjective well-being scale were $0.867,0.943$, and 0.825 , indicating that the reliability of each subscale was high. The KMO value of the index of well-being scale was 0.825 , which was suitable for factor analysis. There were two dimensions extracted, which were overall emotional index and life satisfaction.

\subsection{Descriptive Statistics of Each Scale}

Table2. Descriptive statistics of each variable

\begin{tabular}{|l|l|l|l|l|l|}
\hline & $n$ & Minimum & Maximum & Mean & $S D$ \\
\hline Pressure & 331 & 5.000 & 25.000 & 14.196 & 5.001 \\
\hline Anxiety & 331 & 10.000 & 50.000 & 17.362 & 8.825 \\
\hline Index of well-being & 331 & 13.000 & 63.000 & 43.012 & 9.231 \\
\hline
\end{tabular}

The average score of the returning students on the pressure scale was 14.196 and the standard deviation was 5.001; the average score of the anxiety scale was 17.362 and the standard deviation was 8.825; the average score on the index of well-being scale was 43.012, standard deviation was 9.231.

\subsection{Comparison of the Differences}

Differences analyses were tested in the dimensions of gender, marital status between Pressure, Anxiety and Subjective well-being of returnees, the results were in table 3 and table 4 . 
Table3. Gender differences in Pressure, Anxiety, and Subjective well-being of returning students

\begin{tabular}{|l|l|l|l|}
\hline & $\begin{array}{l}\text { Pressure } \\
(M \pm S \mathrm{D})\end{array}$ & $\begin{array}{l}\text { Anxiety } \\
(M \pm S D)\end{array}$ & $\begin{array}{l}\text { subjective well-being } \\
(M \pm S D)\end{array}$ \\
\hline Male $(n=159)$ & $14.176 \pm 5.290$ & $17.031 \pm 8.767$ & $42.837 \pm 9.137$ \\
\hline Female $(n=172)$ & $14.215 \pm 4.752$ & $17.669 \pm 8.892$ & $43.174 \pm 9.340$ \\
\hline$d f$ & 329 & 329 & 329 \\
\hline$t$ & -0.71 & -.656 & -.333 \\
\hline
\end{tabular}

$* \mathrm{p}<0.05, * * \mathrm{p}<0.01, * * * \mathrm{p}<0.001$

There was no significant difference in the gender dimension of the pressure, anxiety and subjective well-being of returning students.

Table4. Differences in Marital Status between Pressure, Anxiety, and Subjective Well-Being of returning students

\begin{tabular}{|l|l|l|l|}
\hline & Pressure $(M \pm S D)$ & Anxiety $(M \pm S D)$ & subjective well-being $(\mathrm{M} \pm \mathrm{SD})$ \\
\hline $\begin{array}{l}\text { Married } \\
(n=180)\end{array}$ & $12.983 \pm 4.937$ & $15.667 \pm 7.573$ & $44.111 \pm 9.130$ \\
\hline $\begin{array}{l}\text { Unmarried } \\
(n=151)\end{array}$ & $15.642 \pm 4.718$ & $19.384 \pm 9.763$ & $41.702 \pm 9.210$ \\
\hline$d f$ & 329 & 329 & 329 \\
\hline$t$ & -4.980 & $-3.899^{* * * *}$ & 2.382 \\
\hline
\end{tabular}

$* \mathrm{p}<0.05, * * \mathrm{p}<0.01, * * * \mathrm{p}<0.001$

There was no significant difference in the marital status dimension between the pressure status and subjective well-being of returning students; the anxiety status was significantly different in the marital status dimension, and the anxiety level of unmarried people was significantly higher than that of married people.

\subsection{Correlation Analysis of Pressure, Anxiety and Subjective Well-Being of Returning Students}

Table5. Correlation analysis of Pressure, Anxiety and Subjective well-being of returning students

\begin{tabular}{|c|c|c|c|c|}
\hline Variable & $M \pm S D$ & Pressure & Anxiety & subjective well-being \\
\hline Pressure & $14.196 \pm 5.001$ & 1 & & \\
\hline Anxiety & $17.363 \pm 8.825$ & $.482^{* *}$ & 1 & \\
\hline subjective well-being & $43.012 \pm 9.231$ & $-.364^{* *}$ & $-.524^{* *}$ & 1 \\
\hline
\end{tabular}

$* \mathrm{p}<0.05, * * \mathrm{p}<0.01, * * * \mathrm{p}<0.001$

It could be seen from the correlation analysis that the correlation between pressure, anxiety, and subjective well-being of returning students was significant, and that there was a positive correlation between pressure and anxiety, and a negative correlation with subjective well-being. There was a negative correlation.

\subsection{Regression Analysis of Subjective Well-Being of Pressure and Anxiety of Returning Students}

In order to investigate the predictive effects of pressure and anxiety on the subjective well-being of returning students, the pressure and anxiety were used as independent variables, and subjective wellbeing was used as the dependent variable for regression analysis. The results were as follows.

Table6. Regression Analysis of Subjective Well-Being of Pressure and Anxiety of returning students

\begin{tabular}{|c|c|c|c|c|c|c|c|}
\hline Independent variable & Dependent variable & $R$ & $R^{2}$ & $\Delta R^{2}$ & $F$ & $\beta$ & $t$ \\
\hline Pressure & Subjective Well-Being & $.539^{\mathrm{a}}$ & 0.291 & 0.286 & $67.251^{* * *}$ & -0.266 & $-2.720^{* *}$ \\
\hline Anxiety & & & & & & -0.455 & $-8.566^{* * * *}$ \\
\hline
\end{tabular}

$* \mathrm{p}<0.05, * * \mathrm{p}<0.01, * * * \mathrm{p}<0.001$

Regression results showed that the pressure and anxiety of returning students had an effect on subjective well-being. The multivariate correlation coefficient $\mathrm{R}$ was 0.539 and the determination coefficient $\mathrm{R}^{2}$ was 0.286 . This showed that the pressure and anxiety states explained $28.6 \%$ of subjective well-being, and $28.6 \%$ of subjective well-being were caused by pressure and anxiety. 
It could be seen from the coefficient results that the $\mathrm{P}$ value in the regression coefficient of the pressure condition was $<0.01$ and the $\mathrm{P}$ value in the regression coefficient of the anxiety condition was $<0.001$, which indicated that the pressure condition and the anxiety condition had a significant negative impact on subjective well-being, that was, the pressure condition. The higher the state of anxiety, the lower the subjective well-being.

\section{DISCUSSIONS}

\subsection{Pressure, Anxiety, and Subjective Well-Being of Returnees are at a Medium Level}

This study found that pressure, anxiety, and subjective well-being of returnees were all at a medium level. After returning from studying and living abroad, those returning to China did not feel stressful during the adaptation process in society, culture, life, and work. At the same time, their anxiety level remained at a medium level. This is because returnees who have studied abroad have acquired deep subject knowledge and abilities through the study abroad stage, exerted their own advantages in domestic work, and at the same time faced the cultural shock and social environment adaptation problems brought by different environments with an open and inclusive attitude. Properly coped with the adaptation of learning, working, and interpersonal environments at home and abroad, so there is not too much pressure and anxiety; at the same time, the emotional support received by returnees from friends and relatives of the original nationality is positive for their self-adjustment Role (Lore Van Gorpa, SmarandaBoroşb, Piet Brackea\& Peter AJ Stevens, 2017). Surveys show that $80 \%$ of returnees studying abroad believe that the pressure they are currently experiencing is "fully affordable" or "substantially affordable" (Yu Xiaoping, 2016). But at the same time, returnees studying abroad are experiencing the test of problems such as high job expectations and reality, cultural readaptation, and the impact of the domestic system on employment (Li Liping, 2013), so subjective well-being is not very high, and Is maintained at a medium level.

\subsection{The Pressure, Anxiety, and Subjective well-Being of Returnees are not significantly different in the Gender Dimension, and are significantly different in the Marital Status Dimension.}

The pressure, anxiety, and subjective well-being of returnees do not show significant differences in gender. Men's pressure, anxiety, and subjective well-being are the same as those of women. This is because the domestic environment continues to develop towards a more equal direction for men and women. The social environment for men and women gradually tends to be similar or the same, and there is a similar degree of accommodation for individuals of different genders. Therefore, returnees do not feel different because of their gender pressure, anxiety, and subjective well-being.

However, there is a significant difference in the anxiety status of returnees in terms of marital status. Unmarried people experience a more significant level of anxiety than married people. This is because married people can better cope with the problems and challenges brought about by work, life, and social relations after returning to China due to their richer experience and more abundant experience. At the same time, the composition of the family is based on the strong family support of returnees and psychological comfort; unmarried people are relatively young and have little experience, and support from the family is relatively small. They are more likely to be confused and upset when dealing with life and work problems, so they have a higher level of anxiety than married people.

\subsection{Pressure and Anxiety of Returnees are Significantly Negatively Correlated with Subjective Well-Being, and Pressure and Anxiety Negatively Predict Subjective Well-Being}

Correlation analysis and regression analysis of pressure, anxiety, and subjective well-being of returnees showed that pressure, anxiety, and subjective well-being were significantly negatively correlated, and pressure and anxiety had predictive effects on subjective well-being.

This finding is consistent with the findings of Liu Pingping, Tan Lina, and others. Working pressure is positively related to anxiety (Tan Lina, 2015), and pressure has a significant positive predictive effect on anxiety levels (Liu Pingping, 2012). Anxiety, as an important indicator of individual mental health, is closely related to subjective well-being. The study found that employee anxiety is significantly negatively related to subjective well-being. As a common negative emotion, anxiety affects the 
employee's work attitude, reduces work efficiency and affects work-life satisfaction (Liu Lanfei, 2017).

In recent years, due to the pressure of domestic employment, the increase in the number of returnees to the country, the pressure on employment competition, and policy restrictions, the pressure felt by returnees has increased. Pressures such as social and social issues are prone to negative emotions such as doubt, dissatisfaction, and anxiety, which reduce the psychological adaptability of returnees (Shi Xingsong, 2017), as well as the evaluation of life satisfaction and overall well-being

\section{REFERENCES}

[1] Abbott, A., Silles, M. (2016). Determinants of international student migration. The World Economy.39, 621-635. https://doi.org/10.1111/twec. 12319

[2] DuanHongzhen. (2012) Research on Life Satisfaction and Related Factors of Returnees in Beijing [D].Capital Normal University (Master Thesis).

[3] Han Haiping. (2012). A Comparative Study of Subjective Well-being of Returnee Knowledge Workers in Shanghai High-Tech Enterprises. East China Normal University (Master Thesis).

[4] HouRuihe. (2013). Survey of subjective well-being, depression and anxiety among graduate students [J].Chinese Journal of Mental Health,27(6):479-480.

[5] Kahn, S., MacGarvie, M. (2016). Do return requirements increase international knowledge diffusion? Evidence from the Fulbright program. Res. Policy 45, 1304-1322. https://doi.org/10.1016/j.resp ol.2016.02.002

[6] Li Jing. (2008). Cultural Adaptation in the Impact of Reverse Culture_-An Empirical Study of Returned Overseas Chinese Students in Shanghai. Fudan University (Master Thesis).

[7] Li Liping. (2013). Status Quo of Career Development of Returned Overseas Chinese Students and Countermeasures. Southwestern University of Finance and Economics (Master Thesis).

[8] Liu Lanfei. (2017). The Relationship between Workers' Job Burnout and Subjective Well-being — The Mediating Role of Anxiety and Depression [D].Yunnan Normal University (Master Thesis).

[9] Liu Pingping. (2012). The Relationship between Work Stress and Anxiety of Enterprise Technologists [J]. Chinese Journal of Health Psychology, 20 (8): 1172-1175. DOI:10.13342/j.cnki.cjhp.08.003

[10] Lore Van Gorpa, SmarandaBoroşb, Piet Brackea\& Peter AJ Stevens. (2017). Emotional support on re-entry into the home country: Does it matter for repatriates' adjustment who the providers are? International Journal of Intercultural Relations.58: 54-68.

[11] MageswariKunasegaran, Maimunah Ismail, RoziahMohd, Rasdi\&IsmiArifIsmail. (2016). Intercultural and Workplace Adaptation: A Case Study of Malaysian Professional Returnees. Journal of Social and Behavioral Sciences.219, 448-454.

[12] Michel Beine, FrédéricDocquier, Hillel Rapoport. (2001). Brain drain and economic growth: theory and evidence, Journal of Development Economics, Volume 64, Issue 1, 2001, 275-289, https://doi.org/10. 1016/S0304-3878(00)00133-4.

[13] Naito, T., Zhao, L. (2019) .Capital accumulation through studying abroad and return migration, Economic Modelling, Economic Modelling, https://doi.org/10.1016/j.econmod.2019.07.018

[14] Phillip L. Rice. Stress and health. Beijing: China Light Industry Press, 2000.5.

[15] RenHuizhu. (2017). Research on the Relationship between Work Stress, Work Anxiety and Job Happiness of Enterprise Employees [D].Tianjin Normal University (Master Thesis).

[16] Shi Xingsong. (2017). Investigation and Study on the Adaptation of Overseas Returned Teachers in Universities_—A Case Study of Beijing Universities [J].Overseas Chinese History Studies, 2:1-9.

[17] Tan Lina. (2015). A Study on the Relationship between Prison Policewoman's Psychological Resilience and Work Stress, Anxiety and Depression [J].Chinese General Practice,18(28):3481-3484.

[18] TianHaisong., (2012). A Study on the Motivation and Development Factors of Returning High-level Overseas Students_—_Taking Tianjin "Thousand Talents Plan" as an Example [D].Tianjin University(PhD Thesis).

[19] Wu Yanmei., (2013). The Relationship between Subjective Well-being and Performance of Female Overseas Returned Knowledge Workers: An Empirical Study Based on the Technology Enterprise [D].East China Normal University (Master Thesis).

[20] Yin Haoyang. (2018). Research on the Development Status of Returned Students in Guangxi Universities since Reform and Opening Up. Guangxi University for Nationalities (Master Thesis). 
[21] Yu Xiaoping. (2016). Chinese Returnees' Ideological Survey Report [J].People Think Tank, 2017, 01:4043. DOI:10.16619/j.cnki.rmlt.2017.03.020

Citation: Huang Xitong, et.al. "Pressure, Anxiety and Subjective well being in Chinese Overseas Returnees." International Journal of Humanities Social Sciences and Education (IJHSSE), vol 7, no. 3, 2020, pp. 66-72. doi: http://dx.doi.org/10.20431/2349-0381.0703008.

Copyright: (C) 2020 Authors. This is an open-access article distributed under the terms of the Creative Commons Attribution License, which permits unrestricted use, distribution, and reproduction in any medium, provided the original author and source are credited. 